\title{
一般的 $\mathrm{A}_{a} \mathrm{~B}_{b}$ 缩聚反应的固化理论 \\ - II. 高分子矩问题
}

\author{
唐敖庆 李泽生 孙家钟 汤心颔 \\ (吉林大学理论化学研究所、化学系,长葛)
}

㨨 要

本文对一般的 $\mathrm{A}_{a} \mathrm{~B}_{b}$ 缩聚反应的高分子矩进行了研究. 利用我们曾经建议的微 商法,得到了适合于国化全过程的矩的循环公式和 $K$-次矩的解析表示式.

关键词：固化理论，高分子矩， $\boldsymbol{K}$-次矩

\section{一, 引 六}

高分子矩是㩲写高分子固化过程时重要的物理量. 通过它们不仅可以计算出各种平均聚 合度,得到有实际应用价值的凝胶化条件, 而且对于研究溶胶一凝胶相转变过程的标度问题有 着重要意义. 关于高分子矩的计算, Flor $\mathrm{y}^{[1]}$ 和 Stockmayer ${ }^{[2]}$ 曾分别应用母函数的方 法, Gordon ${ }^{[3]}$. Miller 和 Macosko ${ }^{[4,5]}$ 应用概率生成函数的方法有过报道. 本文着重研究一般的 $\mathrm{A}_{a} \mathrm{~B}_{b}$ 缩聚反应的高分子矩问题。一般的 $\mathrm{A}_{\boldsymbol{a}} \mathrm{B}_{b}$ 缩聚反应指的是,单体中含有 $a$ 个 $\mathrm{A}$ 基和 $b$ 个 $\mathrm{B}$ 基,起反应包括 $\mathrm{A}$ 基和 $\mathrm{A}$ 基反应, $\mathrm{B}$ 基和 $\mathrm{B}$ 基反应, $\mathrm{A}$ 基和 $\mathrm{B}$ 基反应. Spouge $\mathrm{e}^{(6)}$ 只研究了 $\mathrm{A}$ 基和 $\mathrm{B}$ 基起反应的 $\mathrm{A}_{a} \mathrm{~B}_{b}$ 缩聚反应. 本文应用文献 $[7,8]$ 曾经建议的微商法，得到了一般 $\mathrm{A}_{a} \mathrm{~B}_{b}$ 缩聚反应的矩的循环公式和 $K$-次矩的解析表示式. 这种微商法的第一个优点是, 不涉 及到分布函数中的组合因子; 第二个优点是,能得到适合于固化全过程的(凝胶点以前和凝胶 点以后)矩的循环公式; 第三个优点是, 这种循环公式的计算, 只涉及到普通的微商运算, 由此 可以得到适合于固化全过程的 $K$-次矩的解析表示式. 上述三个优点是前人方法所不具备的， 因此用他们的方法也就得不到文中的一些结果. 作为本文的特例, 可以得到 Spouge ${ }^{\left[{ }^{[6]}\right.}$ 凝胶 点以前的重均聚合度和凝胶化条件. 关于应用 $K$-次矩的解析表示式开展标度问题的研究, 我 们将另文报道.

\section{二、高分子矩的循环公式}

本节用概率方法, 首先得到一般的 $A_{a} B_{b}$ 缩聚反应的分布函数, 再用微商法得到矩的循环 公式.

令 $i$ 是 $n$-聚物中 $\mathrm{A}$ 基与 $\mathrm{A}$ 基相联之数, $j$ 是 $\mathrm{B}$ 基与 $\mathrm{B}$ 基相联之数, $l$ 是 $\mathrm{A}$ 基与 $\mathrm{B}$ 基視联 本文1988 年12月17日收到。 
之数. 则在 $n$-聚物中, $\mathrm{A}$ 与 $\mathrm{A}$ 相联的 $\mathrm{A}$ 基之数为 $2 i, \mathrm{~B}$ 与 $\mathrm{B}$ 相联的 $\mathrm{B}$ 基之数为 $2 j, \mathrm{~A}$ 与 $\mathrm{B}$ 相联的 $\mathrm{B}$ 基 (或 $\mathrm{A}$ 基)之数为 $l$, 未相联的 $\mathrm{A}$ 基之数为 $a n-2 i-l$,末相联的 $\mathrm{B}$ 基之数为 $b_{n}-$ $2 j-l$. 从概率考虑, 体系中由 $i$ 个 $\mathrm{A}-\mathrm{A}$ 联炶点, $j$ 个 $\mathrm{B}-\mathrm{B}$ 联结点, $l$ 个 $\mathrm{A}-\mathrm{B}$ 联结点构成 的 $n$-聚物数除以体系总单体数 $N$, 即分存函数 $P_{n}(i, j, l)$ 为

$$
\begin{gathered}
P_{n}(i, j, l)=C_{n}(i, j, l, r) P_{a a}^{i} P_{b b}^{\prime} P_{b a}^{l}\left(1-P_{a b}-P_{a b}\right)^{a n-2 i-l} \\
\cdot\left(1-P_{b b}-P_{b a}\right)^{b n-2 j-l},
\end{gathered}
$$

式中 $P_{a a}, P_{a b}, P_{b b}, P_{b a}$ 分别代表体系中 $\mathrm{A}$ 基与 $\mathrm{A}$ 基, $\mathrm{A}$ 基与 $\mathrm{B}$ 基, $\mathrm{B}$ 基与 $\mathrm{B}$ 基, $\mathrm{B}$ 基与 $\mathrm{A}$ 基 的总反应程度,式中的 $C_{x}(i, j, l, r)$ 为分布函数的组合因子,其中 $r$ 为

$$
r=\frac{b}{a},
$$

特别要注意到,任何反应类型的分布函数中的组合因子,在原则上都可以得到. 但用微商法推 导矩的循环公式, 则完全不需要知道组合因子的具体表示式, 这也是我们建议微商法的优点之

在 $n$-聚物中, 共有 $n-1$ 个联结点, 因此分布中的指标 $i, j, l, n$ 满足

$$
i+j+l=n-1,
$$

根据分布函数 $P_{n}(i, j, l, r)$ 的物理意义, 对分布函数中的指标 $i, i, l$ 求和, 求和指标满足 $i+j+l=n-1$, 则

$$
P_{n}=\sum_{\substack{i, j, l \\ i+i+l=n-1}} P_{n}(i, j, l),
$$

代表体系中总的 $n$-聚物数 (不管各种联结点怎样分布) 除以 $N$.

由 (4) 式,就可以写出一般 $\mathrm{A}_{a} \mathrm{~B}_{b}$ 缩聚反应的 $K$-次矩表示式

$$
M_{K}=\sum_{n} n^{K} P_{s}=\sum_{n} \sum_{\substack{i, j, l \\ i+j+l=n-1}} n^{K} P_{n}(i, i, l),
$$

将分布函数 (1) 式代人上式,得

$$
\begin{aligned}
M_{K}= & \sum_{n} \sum_{\substack{i, j, l \\
i+i+l=n-1}} n^{K} C_{n}(i, j, l, r) P_{a a}^{i} P_{b b}^{i} P_{b a}^{l}\left(1-P_{a s}-P_{a b}\right)^{a n-2 i-l} \\
& \cdot\left(1-P_{b b}-P_{b a}\right)^{b n-2 j-l},
\end{aligned}
$$

上式有 4 个求和指标 $n, i, j, l$, 由于约束条件 $i+j+l=n-1$, 因此只有 3 个独立求和指 标. 在分布函数中, 有 4 个物理量 $P_{a a}, P_{a b}, P_{b b}, P_{b a}$, 由于存在 $a P_{a b}=b P_{b a}$ 关系, 只有 3 个物 理量是独立变量. 本文选 $P_{a a}, P_{b b}, P_{b a}$ 作为独立变量. 为了推导矩的循环公式, 先引进两个 垔 $R_{K}$ 和 $T_{K}$ 。

$$
\begin{aligned}
& R_{K}=\sum_{n} \sum_{\substack{i, j, l \\
1+j+l=n-1}} i{ }^{K} P_{n}(i, j, l), \\
& T_{K}=\sum_{n} \sum_{\substack{i, j, l \\
i+i+l=n-1}} i n^{K} P_{n}(i, j, l) .
\end{aligned}
$$

利用 (7) 和 (8) 式, 以及限制条件 $i+j+l=n-1$, 从 $K$-次矩的表示式 (6) 式, 可得如下 关系式 


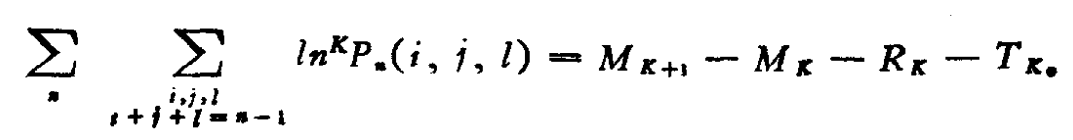

对 $K$-次矩表示式 (6) 式左右两端分别对 $P_{a a}, P_{b b}, P_{b a}$ 进行偏微商, 这一过程不涉及到: 组合因子的具体表示式, 再利用 (7), (8) 和 (9) 式, 可得

$$
\begin{gathered}
\left(1-P_{a b}\right) R_{K}-(a-1) P_{a a} M_{K+1}-P_{a a} T_{K}=P_{a a}\left(1-P_{a a}-P_{a b}\right) \frac{\partial M_{K}}{\partial P_{a b}}+P_{a a} M_{K}, \\
-P_{b b} R_{K}-(b-1) P_{b b} M_{K+1}+\left(1-P_{b a}\right) T_{K}=P_{b b}\left(1-P_{b b}-P_{b a}\right) \frac{\partial M_{K}}{\partial P_{b b}}+P_{b b} M_{K} \\
-P_{b b}\left(P_{a a}+P_{a b}\right) R_{K}+P_{a a} P_{b b} M_{K+1}-P_{a c}\left(P_{b b}+P_{b a}\right) T_{K}=P_{a a} P_{b b}\left(P_{b a} \frac{\partial M_{K}}{\partial P_{b a}}\right. \\
\left.-P_{a b} \frac{\partial M_{K}}{\partial P_{a c}}-P_{b a} \frac{\partial M_{K}}{\partial P_{b b}}+M_{K}\right) .
\end{gathered}
$$

在以上 3 个方程式中, 有 3 个未知量 $R_{\mathrm{K}}, M_{\mathrm{X}+1}$ 和 $T_{K}$, 求解方程组, 可以得到它们的解. 本 文只需要 $M_{K+1}$ 的解, 其它 2 个解省略, 即

$$
M_{K+1}=\frac{1}{D^{0}-D}\left(E M_{K}+F \frac{\partial M_{K}}{\partial P_{a}}+I \frac{\partial M_{K}}{\partial P_{b b}}+J \frac{\partial M_{K}}{\partial P_{b a}}\right),
$$

上式的右方涉及到 $K$-次矩 $M_{K}$,左方是 $(K+1)$-次矩, 因此 (13) 式就是矩的循环公式. 式 中

$$
\begin{aligned}
& D^{0}=1 \\
& D=2 a P_{a b}+(a-1) P_{a a}+(b-1) P_{b b}+(a+b-1) P_{a c} P_{b b}-(a+b-1) P_{a b} P_{b a}, \\
& E=1+P_{a a}+P_{b b}+P_{a a} P_{b b}-P_{a b} P_{b a} \\
& F=P_{a a}\left[\left(1-P_{a b}\right)\left(1+P_{b b}\right)-P_{a b}\left(1-P_{b a}\right)-P_{a b}\right] \\
& I=P_{b b}\left[\left(1-P_{b b}\right)\left(1+P_{a c}\right)-P_{b a}\left(1-P_{a b}\right)-P_{b a}\right] \\
& J=P_{b a}\left[\left(1-P_{a b}\right)\left(1-P_{b a}\right)-P_{a c} P_{b b}\right] .
\end{aligned}
$$

显然, 矩的循环公式适用于固化的全过程. 如果 $K$-次矩为已知, 则可以利用循环公式推导 $(K+1)$-次矩, 其运算过程只涉及到普通的微商运算.

\section{三、高分子 $\boldsymbol{K}$-次矩的解析表示式}

本节将应用矩的很环公式, 进一步推导 $K$-次矩的解析表示式, 其结果适用于固化的全过 程. 得到这一解析表示式, 可直接用来讨论各种平均聚合度的计算. 在另一篇论文中将会看 到, 它和固化中溶胶一凝胶相转变的标度问题密切相关.

当 $K=0$ 时,由 (5) 式所定义的零次矩为

$$
M_{0}=\sum_{n} \sum_{\substack{i, j, l \\ i+i+i=n-1}} P_{n}(i, j, l),
$$

宙其中分布函数的物理意义, 可以直接得到 $M_{0}$ 的物理意义为溶胶中高分子数目除以 $N$. 在 栨胶点以前, 体系没有凝胶。当没有起反应时, 体系中有 $N$ 个单体. 当起反应时, 每形成一个 
聐点, 体系中减少一个单体, 也就是体系中高分子数等于 $N$ 减去联结点数. 由于 $\mathrm{A}$ 基与 $\mathrm{A}$ 基的联结点数为 $a N P_{a s} / 2, \mathrm{~B}$ 基与 $\mathrm{B}$ 基的联结点数为 $b N P_{b b} / 2, \mathrm{~A}$ 基与 $\mathrm{B}$ 基的联结点数为 $a N P_{a b}=b N P_{b a}=\left(a N P_{a b}+b N P_{b a}\right) / 2$, 因此凝胶点以前的零次矩可表示为 $1-a\left(P_{a a}+P_{a b}\right) /$ $2-b\left(P_{b b}+P_{b a}\right) / 2$. 在凝胶点以后, 体系出现凝胶. 溶胶中有 $N^{\prime}=N S$ 个单体, 其中 $S$ 为 溶胶分数. 在溶胶中, 每形成一个联结点, 溶胶中减少一个单体. 也就是溶胶中的高分子个数 等于 $N^{\prime}$ 减去联结点数. 在溶胶中, $\mathrm{A}$ 基与 $\mathrm{A}$ 基的联结点数为 $a N^{\prime} P_{a_{a}}^{\prime} / 2, \mathrm{~B}$ 基与 $\mathrm{B}$ 基的联结点 数为 $b N^{\prime} P_{b b}^{\prime} / 2, \mathrm{~A}$ 基与 $\mathrm{B}$ 基的联结点数为 $a N^{\prime} P_{a b}^{\prime}=b N^{\prime} P_{b a}^{\prime}=\left(a N^{\prime} P_{a b}^{\prime}+b N^{\prime} P_{b a}^{\prime}\right) / 2$, 因此凝胶 点以后的零次矩可以表示为 $S\left[1-a\left(P_{a b}^{\prime}+P_{a b}^{\prime}\right) / 2-b\left(P_{b b}^{\prime}+P_{b a}^{\prime}\right) / 2\right]$, 其中 $P_{a a}^{\prime}, P_{a b}^{\prime}, P_{b b}^{\prime}, P_{b a}^{\prime}$ 分别代表溶胶中 $\mathrm{A}$ 基与 $\mathrm{A}$ 基, $\mathrm{A}$ 基与 $\mathrm{B}$ 基, $\mathrm{B}$ 基与 $\mathrm{B}$ 基, $\mathrm{B}$ 基与 $\mathrm{A}$ 基的反应程度. 总之, 零次矩 $M$ 。可以表示为

$$
M_{0}=\left\{\begin{array}{l}
1-\frac{a\left(P_{a b}+P_{a b}\right)}{2}-\frac{b\left(P_{b b}+P_{b c}\right)}{2}, \text { 凝胶点以前, } \\
S\left[1-\frac{a\left(P_{a b}^{\prime}+P_{a b}^{\prime}\right)}{2}-\frac{b\left(P_{b b}^{\prime}+P_{b a}^{\prime}\right)}{2}\right], \text { 凝胶点以后. }
\end{array}\right.
$$

在文献 [7] 中, 溶胶分数 $S$ 可表示为

$$
\begin{aligned}
& S=X^{a} Y^{b}, \\
& X=1-P_{a c}-P_{a b}+P_{a b} X^{a-1} Y^{b}+P_{a b} X^{a} Y^{b-1}, \\
& Y=1-P_{b b}-P_{b a}+P_{b b} X^{a} Y^{b-1}+P_{b a} X^{a-1} Y^{b} .
\end{aligned}
$$

溶胶反应程度和总反应程度有如下关系

$$
\begin{aligned}
& P_{a b}^{\prime}+P_{a b}^{\prime}=1-\frac{1-P_{a a}-P_{a b}}{X}, \\
& P_{b b}^{\prime}+P_{b a}^{\prime}=1-\frac{1-P_{b b}-P_{b a}}{Y} .
\end{aligned}
$$

将 $M_{0}$ 作为起始点,利用循环公式 (13) 式, 1 次矩 $M_{1}$ 可以表示为

$$
M_{1}=\frac{1}{D^{6}-D}\left[E M_{0}+F \frac{\partial M_{0}}{\partial P_{a c}}+I \frac{\partial M_{0}}{\partial P_{b b}}+J \frac{\partial M_{0}}{\partial P_{b c}}\right] \text {, }
$$

将 $M_{0}$ 的表示式代人上式右方, 可知会遇到一些具体的微商运算, 如

$$
\frac{\partial S}{\partial P_{x x}}, \frac{\partial\left(P_{a c}^{\prime}+P_{a b}^{\prime}\right)}{\partial P_{x x}}, \frac{\partial\left(P_{b b}^{\prime}+P_{b c}^{\prime}\right)}{\partial P_{x x}},
$$

其中 $P_{x x}=P_{a a}, P_{b b},, P_{b a b}$ 为了便于读者实际应用,下面给出它们微商的表示式:

$$
\begin{aligned}
& \frac{\partial S}{\partial P_{x x}}=X^{a-1} Y^{b-1}\left(a Y \frac{\partial X}{\partial P_{x x}}+b X \frac{\partial Y}{\partial P_{x x}}\right), \\
& \frac{\partial\left(P_{a a}^{\prime}+P_{a b}^{\prime}\right)}{\partial P_{x x}}=\frac{1}{X} \frac{\partial\left(P_{a c}+P_{a b}\right)}{\partial P_{x x}}+\frac{\left(1-P_{a a}-P_{b a}\right)}{X^{2}} \frac{\partial X}{\partial P_{x x}}, \\
& \frac{\partial\left(P_{b b}^{\prime}+P_{b a}^{\prime}\right)}{\partial P_{x x}}=\frac{1}{Y} \frac{\partial\left(P_{b b}+P_{b a}\right)}{\partial P_{x x}}+\frac{\left(1-P_{b b}-P_{b a}\right)}{Y^{2}} \frac{\partial Y}{\partial P_{x x}},
\end{aligned}
$$

在推导上式过程中用到了 (22), (25) 和 (26) 式. 从 (28)-(30) 式可以看出,其中的

$$
\frac{\partial\left(P_{a c}+P_{a b}\right)}{\partial P_{x x}} \text { 和 } \frac{\partial\left(P_{b b}+P_{b a}\right)}{\partial P_{x x}}
$$


很容易计算, 只需进一步得到 $\frac{\partial X}{\partial P_{x x}}$ 和 $\frac{\partial Y}{\partial P_{x x}}$ 的微商结果. 利用 (23) 式和 (24) 式对 $P_{a a}$ 偏微 商, 可得

$$
\begin{gathered}
{\left[a\left(1-P_{a b}-P_{a b}\right)+P_{a a} X^{a-1} Y^{b}-(a-1) X\right] \frac{1}{X} \frac{\partial X}{\partial P_{a b}}+\left[b\left(1-P_{a a}-P_{a b}\right)\right.} \\
\left.+P_{a b} X^{a} Y^{b-1}-b X\right] \frac{1}{Y} \frac{\partial Y}{\partial P_{a c}}=X^{a-1} Y^{b}-1, \\
{\left[a\left(1-P_{b b}-P_{b a}-Y\right)+P_{b a} X^{a-1} Y^{b}\right] \frac{1}{X} \frac{\partial X}{\partial P_{a c}}+\left[b\left(1-P_{b b}-P_{b a}\right)\right.} \\
\left.+P_{b b} X^{a} Y^{b-1}\right] \frac{1}{Y} \frac{\partial Y}{\partial P_{a a}}=0
\end{gathered}
$$

以上两式中, $\frac{\partial X}{\partial P_{\text {og }}}$ 和 $\frac{\partial Y}{\partial P_{\text {c。 }}}$ 为末知量, 求解得

$$
\begin{aligned}
& \frac{\partial X}{\partial P_{a} a}=\frac{X}{\Delta}\left(X^{a-1} Y^{b}-1\right)\left[P_{b b} X^{a} Y^{b-1}+b\left(1-P_{b b}-P_{b a}-Y\right)+Y\right], \\
& \frac{\partial Y}{\partial P_{a b}}=\frac{Y}{\Delta}\left(1-X^{a-1} Y^{b}\right)\left[P_{b b} X^{a-1} Y^{b}+a\left(1-P_{b b}-P_{b a}-Y\right)\right],
\end{aligned}
$$

式中

$$
\begin{aligned}
\Delta= & \left(P_{a} P_{b b}-P_{a b} P_{b a}\right) X^{2 a-1} Y^{2 b-1}-(a-1) P_{b b} X^{a+1} Y^{b-1}-(b-1) \\
& +P_{a a} X^{a-1} Y^{b+1}+a\left[\left(1-P_{a b}-P_{a b}\right) P_{b b}-\left(1-P_{b b}-P_{b a}\right) P_{a b}\right] X^{a} Y^{b-1} \\
& +b\left[\left(1-P_{b b}-P_{b a}\right) P_{a b}-\left(1-P_{a c}-P_{a b}\right) P_{b a}\right] X^{a-1} Y^{b}+2 a_{a b} X^{a} Y^{b} \\
& +(1-a-b) X Y+b\left(1-P_{b b}-P_{b a}\right) X+a\left(1-P_{a c}-P_{a b}\right) Y,
\end{aligned}
$$

同理, (23) 和 (24) 式两边分别做 $P_{b b}$ 和 $P_{b a}$ 的偏微商, 可得

$$
\begin{aligned}
\frac{\partial X}{\partial P_{b b}}= & \frac{X}{\Delta}\left(1-X^{a} Y^{b-1}\right)\left[b\left(1-P_{a a}-P_{a b}-X\right)+P_{a b} X^{a} Y^{b-1}\right], \\
\frac{\partial Y}{\partial P_{b b}}= & \frac{Y}{\Delta}\left(X^{a} Y^{b-1}-1\right)\left[a\left(1-P_{a a}-P_{a b}-X\right)+P_{a a} X^{a-1} Y^{b}+X\right], \\
\frac{\partial X}{\partial P_{b}}= & \frac{X}{\Delta}\left[r\left(X^{a} Y^{b-1}-1\right)\left(P_{b b} X^{a} Y^{b-1}+b\left(1-P_{b b}-P_{b a}-Y\right)+Y\right)\right. \\
& \left.+\left(1-X^{a-1} Y^{b}\right)\left(P_{a b} X^{a} Y^{b-1}+b\left(1-P_{a c}-P_{a b}-X\right)\right)\right], \\
\frac{\partial Y}{\partial P_{b a}}= & \frac{Y}{\Delta}\left[\left(X^{a-1} Y^{b}-1\right)\left(P_{a b} X^{a-1} Y^{b}+a\left(1-P_{a c}-P_{a b}-X\right)+X\right)\right. \\
& \left.+r\left(1-X^{a} Y^{b-1}\right)\left(P_{b a} X^{a-1} Y^{b}+a\left(1-P_{b b}-P_{b a}-Y\right)\right)\right] .
\end{aligned}
$$

将 (33)，(34)，(35)-(39) 式代人 (28), (29) 和 (30) 式, 然后代入 (27) 式, 可得

$$
M_{1}=\left\{\begin{array}{l}
1, \text { 凝胶点以前, } \\
s, \text { 凝胶点以后. }
\end{array}\right.
$$

$M_{1}$ 也可由分布函数的物理意义直接得到以上的结果. 这样, 整个对矩的理论就自洽了. 利用 1 次矩 $M_{1}$, 通过循环公式, 可得到 2 次矩 $M_{2}$ 为 


$$
M_{2}=\left\{\begin{array}{l}
\frac{V_{2}}{D^{0}-D}, \text { 凝胶点以前, } \\
\frac{T_{2}}{D-D^{0}}, \text { 凝胶点以后, }
\end{array}\right.
$$

式中

$$
\begin{aligned}
& V_{2}=E, \\
& T_{2}=-\left(E S+F \frac{\partial S}{\partial P_{a b}}+l \frac{\partial S}{\partial P_{b b}}+J \frac{\partial S}{\partial P_{b a}}\right),
\end{aligned}
$$

其中微商 $\frac{\partial S}{\partial P_{x x}}, P_{x x}=P_{a s}, P_{b b}, P_{b c}$ 的结果, 已由 (28), (33), (34), (36)-(39) 式给出.

从 2 次矩 $M_{2}$, 利用循环公式, 可得 3 次矩. 依次循环, 所得 $K$-次矩 $(K \geqslant 2)$ 的解析表 示式为

$$
\begin{aligned}
M_{K} & =\sum_{n} n^{K} P_{n}, \\
& =\sum_{n} \sum_{\substack{i, j, l \\
i+i=n-1 \\
i+l=n}} n^{K} P_{n}(i, i, l), \\
& =\left\{\begin{array}{l}
\frac{V_{K}}{\left(D^{0}-D\right)^{2 K-3}}, \\
\frac{T_{K}}{\left(D-D^{0}\right)^{2 K-3}}, \text { 凝胶点以前, }
\end{array}\right.
\end{aligned}
$$

式中 $V_{K}$ 和 $T_{K}$ 满足同一个循环公式:

$$
\begin{aligned}
W_{K}= & (2 K-5) H W_{K_{-j}}+\left(D^{0}-D\right)\left(E W_{K-1}+F \frac{\partial W_{K_{-1}}}{\partial P_{a c}}\right. \\
& \left.+I \frac{\partial W_{K-1}}{\partial P_{b b}}+J \frac{\partial W_{K_{-1}}}{\partial P_{b c}}\right)
\end{aligned}
$$

其中

$$
\begin{gathered}
H=F\left[a-1+(a+b-1) P_{b b}\right]+I\left[b-i+(a+b-1) P_{a c}\right] \\
+2 J\left[b-(a+b-1) P_{a b}\right], \\
W_{K}=\left\{\begin{array}{l}
V_{K}, \text { 凝胶点以前, } \\
T_{K}, \text { 凝胶点以后. }
\end{array}\right.
\end{gathered}
$$

(45) 式中遇到微瓦 $\frac{\partial W_{K-1}}{\partial P_{x x}}, P_{x x}=P_{a a}, P_{b b}, P_{b a}$ 的计算, 因为 $M_{2}$ 中的微商已经得到. 利 用它们, 依次循环, 就可以计算出所需要的微商.

(44)式为一般的 $\mathrm{A}_{a} \mathrm{~B}_{b}$ 缩聚反应的 $K$-次矩 $(K \geqslant 2)$ 的解析表示式. 利用它很容易计算 客种平均聚合度。例如

数均聚合度

$$
D P_{k}=\frac{M_{1}}{M_{0}}=\left\{\begin{array}{l}
{\left[1-\frac{a\left(P_{a b}+P_{a b}\right)}{2}-\frac{b\left(P_{b b}+P_{b b}\right)}{2}\right]^{-1}, \text { 凝胶点以前, }} \\
{\left[1-\frac{a\left(P_{a b}^{\prime}+P_{b b}^{\prime}\right)}{2}-\frac{b\left(P_{b b}^{\prime}+P_{b c}^{\prime}\right)}{2}\right]^{-1}, \text { 凝胶点以后. }}
\end{array}\right.
$$


重均聚合度

$$
D P_{W}=\begin{aligned}
& M_{2} \\
& M_{1}
\end{aligned}=\left\{\begin{array}{l}
\frac{V_{2}}{D^{0}-D}, \text { 凝胶点以前, } \\
\frac{T_{2}}{S\left(D-D^{0}\right)}, \text { 凝胶点以后. }
\end{array}\right.
$$

$\boldsymbol{Z}$-坞繁合度

$$
D P_{i}=\frac{M_{3}}{M_{2}}=\left\{\begin{array}{c}
\frac{V_{3}}{V_{2}\left(D^{0}-D\right)^{2}}, \\
\frac{T_{3}}{T_{2}\left(D-D^{0}\right)^{2}},
\end{array}\right. \text { 凝胶胶点以前, }
$$

数均、重均聚合度已由 (48) 和 (49) 式给出解析表示式. $Z$-均聚合度中的 $V_{3}$ 和 $T_{3}$, 可利用 $V_{2}$ 和 $T_{2}$ 的表示式,利用循环公式 (45) 就可算出.

Spouge ${ }^{[6]}$ 曾讨论了只有 $\mathrm{A}$ 和 B 基起反应的特殊情况,得到了凝胶点以前的重均聚合度. 作为 (49) 式的特例, 只要令凝胶点以前的 $V_{2}$ 和 $D^{0}-D$ 中的 $P_{a a}$ 和 $P_{b b}$ 为零, 即可得到它的 结果

$$
D P_{W}=\frac{1-P_{a b} P_{b a}}{\left(1-a P_{a b}\right)^{2}-(a-1)(b-1) P_{a b} P_{b a}} \text {, 促胶点以前. }
$$

从重均聚合度表示式 (49), 可进一步得到凝胶化条件,也即当

$$
D^{0}-D=0
$$

时,重均聚合度变为无穷大, 这时有溶胶一凝胶相转变发生,因此 (52) 式就是凝胶化条件. 它 可具体表为

$$
\begin{aligned}
1= & 2 a P_{a b}+(a-1) P_{a a}+(b-1) P_{b b}+(a+b-1) P_{a a} P_{b b} \\
& -(a+b-1) P_{a b} P_{b a},
\end{aligned}
$$

上式与文献 [7] 中, 从溶胶一凝胶分配公式直淁得到的凝胶化条件一致。当只有 $\mathrm{A}$ 基和 $\mathrm{B}$ 基反 应时, 即 $P_{a z}=P_{b b}=0$, 从 (53) 式也可得到唐教庆 ${ }^{[0]}$ 和 $S p o u g \mathrm{e}^{[6]}$ 他们曾分别得到的结果.

\section{参考文献}

[1] Flory, P. J., J. Am. Chem. Soc, 63(1941), 3038, 3091, 3096.

[2] Stockmayer, W. H., J. Chem. Phys., 11(1943), 45.

[3] Gordon, M \& Scantlebury, G., J. Chem. Soc., 1967, 1.

[ 4] Macosko, C. W. \& Miller, D. R., Macromol, 9(1976), 199.

[5] Miller, D. R. \& Macosko, C. W. ibid, 9(1976), 206.

[6] Spouge, L. J., ibid., 16(1983), 121, 831.

[7] Tang Au-chin et al., J. Macromul. Sci-Chem., A25(1988), 41.

[8] Tang Au-Chin et al., Macromol, 21(1988), 797. 